\title{
Systems Analysis of In-Space Manufacturing Applications for the International Space Station and the Evolvable Mars Campaign
}

\author{
Andrew C. Owens* and Olivier L. de Weck ${ }^{\dagger}$ \\ Massachusetts Institute of Technology, Cambridge, MA 02139, USA
}

\begin{abstract}
Maintenance logistics support is a significant challenge for extended human operations in space, especially for missions beyond Low Earth Orbit (LEO). For missions to Mars (such as NASA's Evolvable Mars Campaign (EMC)), where timely resupply or abort in the event of emergency will not be possible, maintenance logistics mass is directly linked to the Probability of Loss of Crew ( $\mathrm{P}(\mathrm{LoC}))$, and the cost of driving down risk is an exponential increase in mass requirements. The logistics support strategies that have maintained human operations in LEO will not be effective for these deep space missions. In-Space Manufacturing (ISM) is a promising technological solution that could reduce logistics requirements, mitigate risks, and augment operational capabilities, enabling Earthindependent human spaceflight. This paper reviews maintenance logistics challenges for spaceflight operations in LEO and beyond, and presents a summary of selected results from a systems analysis of potential ISM applications for the ISS and EMC. A quantitative modeling framework and sample assessment of maintenance logistics and risk reduction potential of this new technology is also presented and discussed.
\end{abstract}

\section{Nomenclature}

$\begin{array}{ll}\text { 3DP } & \text { 3D Printing in Zero-G } \\ \text { AES } & \text { Advanced Exploration Systems } \\ \text { AM } & \text { Additive Manufacturing } \\ \text { AMF } & \text { Additive Manufacturing Facility } \\ \text { CDF } & \text { Cumulative Distribution Function } \\ \text { CDRA } & \text { Carbon Dioxide Removal Assembly } \\ \text { CRA } & \text { Carbon Dioxide Reduction Assembly } \\ \text { DARPA } & \text { Defense Advanced Research Projects Agency } \\ \text { DSH } & \text { Deep Space Habitat } \\ \text { ECLS } & \text { Environmental Control and Life Support } \\ \text { EDL } & \text { Entry, Descent, and Landing } \\ \text { EMC } & \text { Evolvable Mars Campaign } \\ \text { FCPA } & \text { Fluids Control and Pump Assembly } \\ \text { HEOMD } & \text { Human Exploration and Operations Mission Directorate } \\ \text { ISM } & \text { In-Space Manuacturing } \\ \text { ISRU } & \text { In-Situ Resource Utilization } \\ \text { ISS } & \text { International Space Station }\end{array}$

*Ph.D. Candidate and NASA Space Technology Research Fellow, Department of Aeronautics and Astronautics, 77 Massachusetts Ave. Bldg. 33-409, AIAA Student Member.

${ }^{\dagger}$ Professor of Aeronautics and Astronautics and Engineering Systems, Department of Aeronautics and Astronautics, 77 Massachusetts Ave. Bldg. 33-410, AIAA Associate Fellow. 


$\begin{array}{ll}\text { IVA } & \text { Intravehicular Maintenance } \\ \text { LEO } & \text { Low Earth Orbit } \\ \text { MEL } & \text { Master Equipment List } \\ \text { MTBF } & \text { Mean Time Between Failures } \\ \text { NRC } & \text { National Research Council } \\ \text { OGA } & \text { Oxygen Generation Assembly } \\ \text { P }(\text { LoC }) & \text { Probability of Loss of Crew } \\ \text { P }(\text { LoM }) & \text { Probability of Loss of Mission } \\ \text { PMF } & \text { Probability Mass Function } \\ \text { POS } & \text { Probability of Sufficiency } \\ \text { PRA } & \text { Probabilistic Risk Assessment } \\ \text { UPA } & \text { Urine Processor Assembly } \\ \text { WPA } & \text { Water Processor Assembly }\end{array}$

\section{Introduction}

GXTended human operations in space - both in Low Earth Orbit (LEO) and beyond - require significant Elogistics support. On the International Space Station (ISS), spare parts, tools, consumables, and other useful components are periodically shipped from the Earth into space aboard a diverse fleet of government and commercial resupply vehicles. For human missions to Mars, such as those described by NASA's Evolvable Mars Campaign (EMC), periodic resupply of this kind will not be possible. Longer mission endurances increase the probability that failures will occur, and therefore increase the logistics mass that must be carried to account for them. In addition, the lack of timely abort options on these Mars missions that would return the crew safely to the Earth in case of an emergency (options which are available on the ISS) increases the criticality of various system failures. Overall, logistics represent a significant operational expense for the ISS and a major challenge to safe and affordable human exploration beyond ISS. ${ }^{1-5}$

In-Space Manuacturing (ISM) is a promising technological solution that could reduce the logistics footprint of human operations in space, reduce risks, and augment operational capabilities beyond that which can be achieved with a purely Earth-based supply chain. Recent technological advances in fields such as Additive Manufacturing (AM) have resulted in compact, self-contained manufacturing systems that can be deployed within a spacecraft environment to produce useful components in-situ, using raw materials that could be launched from Earth, recycled from obsolete or failed components, or produced using In-Situ Resource Utilization (ISRU) technology. ${ }^{6}$ The 3D Printing in Zero-G (3DP) technology demonstration mission on the ISS has demonstrated ISM technology in an orbital environment, and ongoing technology development will expand manufacturing capabilities in terms of materials selection, precision, size, and complexity, among others. $^{7,8}$ The development of new capabilities and the design of systems and mission plans that exploit these new capabilities must be guided by holistic systems analysis that considers cost, risk, and performance impacts in terms of mission-level metrics. The examination and modeling of the impacts of ISM in a systems context allows its consideration in system trade studies, which can then inform technology, system, and mission development efforts. ${ }^{9}$

This paper presents a summary of selected results from a systems analysis of potential ISM applications for the ISS and EMC performed in support of the Human Exploration and Operations Mission Directorate (HEOMD) Advanced Exploration Systems (AES) ISM Project. The remainder of this paper is organized as follows. Section II presents an overview and discussion of the maintenance logistics and risk challenges of spaceflight, including experience and lessons learned from ISS operations and their implications for future deep space missions. Section III presents a brief overview of proposed applications of ISM in spaceflight, along with a discussion of their potential impacts. Section IV presents a quantitative modeling approach to asses logistics requirements with and without ISM and/or materials recycling, including assessment of the impact of parameter uncertainty. A case study is presented that examines the trade between maintenance logistics and risk for the EMC Deep Space Habitat (DSH) on a Mars transit mission and how it is impacted by ISM. Section V discusses the results of the case study presented in section IV as well as more qualitative considerations described in sections II and III, and section VI summarizes the results of this paper and presents conclusions. 


\section{Maintenance Logistics and Risk Challenges}

All human spaceflight experience has been relatively close to home, and has made use of regular resupply opportunities and quick abort options to mitigate risk associated with maintenance. The Apollo missions, the most distant human spaceflight missions to date, were all short-duration, and had the option to return to Earth in a matter of days in the event of an emergency. This abort capability enabled the safe return the crew of Apollo 13 after catastrophic failure on board their spacecraft. More recently, long-duration human spaceflight on the ISS has been supported by on-board storage of a large number of spares, regular resupply missions from Earth, and from LEO the crew have the ability to abort and return to Earth within hours. Future missions will send humans farther from Earth than they have ever gone before, and they will remain in deep space - beyond the reach of timely resupply and without the option of a quick abort home - for significantly longer than previous missions. The lack of abort and resupply options for deep space missions presents a very significant logistics and risk challenge for future missions, and creates a situation that has never before been encountered in human spaceflight. ${ }^{1-3,10}$

\section{A. Duration vs. Endurance}

Here it is important to distinguish between long-duration spaceflight and long-endurance spaceflight. Duration refers to the time between mission start and end, and in the human spaceflight context is often used to refer to the total amount of time the crew spends in space, or the amount of time that the ISS is continuously occupied. Endurance, on the other hand, represents the amount of time that a system must go without resupply. ${ }^{11}$ For many human spaceflight missions, such as Apollo and Shuttle, duration and endurance are equivalent - and both are short. For the ISS, however, endurance is much shorter than duration due to the regular resupply flights from the ground. Though crews have maintained a presence in space on the ISS for a decade and a half, and some have spent nearly a year in space, all of these missions were supported by resupply flights that occurred every few months. As a result, these long-duration missions have a short endurance.

This distinction between duration and endurance is important because endurance is the driving factor for maintenance logistics requirements, not duration. When a failure occurs, there is typically some time to impact between the actual time of failure and the time at which the impact of that failure becomes critical. For short-endurance missions, or missions with abort and on-demand resupply opportunities, this time between failure occurrence and critical impact can allow the use of these options to mitigate risk to the crew. For long-endurance missions, however, the lack of abort and resupply options (or severely limited options, if they exist) may mean that the time between failure occurrence and critical impact may be shorter than the time required to use these options. As a result, the criticality of failures on long-endurance missions is increased. Humans have accumulated long-duration spaceflight experience, but have not yet experienced long-endurance spaceflight on the scales that will be required to go to Mars and back. The EMC DSH, for example, must be able to support the crew for 1,100 days in space without resupply for a Mars transit mission (round trip, including time in Mars orbit as a safe haven), ${ }^{12}$ over 12 times longer than the typical 3 month resupply interval for the ISS.

\section{B. ISS Experience and Implications for Future Missions}

\section{High Maintenance Mass Requirement}

Even in LEO, with ready access to the ground for logistics support and quick abort options, the ISS requires a very significant amount of maintenance logistics mass. Figure 1 shows the estimated ISS maintenance logistics requirements for 2012-2020, calculated by Cirillo et al. ${ }^{2}$ using predicted Mean Time Between Failures (MTBF) data from ISS estimates in 2011. Over 13,000kg of on-orbit spares are provisioned to cover potential failures, and nearly $18,000 \mathrm{~kg}$ are kept ready on the ground, to be flown if necessary; in total, over $31,000 \mathrm{~kg}$ of spares are kept available to respond to maintenance demands. ${ }^{2}$ A small number of very large items does drive this mass upwards, and not all items that are spared for on the ISS will be carried on a Mars transit mission. However, figure 1 is indicative of the current state of maintenance logistics supply, and provides context for the challenges that will be faced in the future.

High logistics mass alone is a significant issue for future missions, but figure 1 also illustrates one of the primary challenges of maintenance logistics: though a large amount of spares mass is required to cover potential failures, only a relatively small amount of that mass is expected to actually be used. This is a 


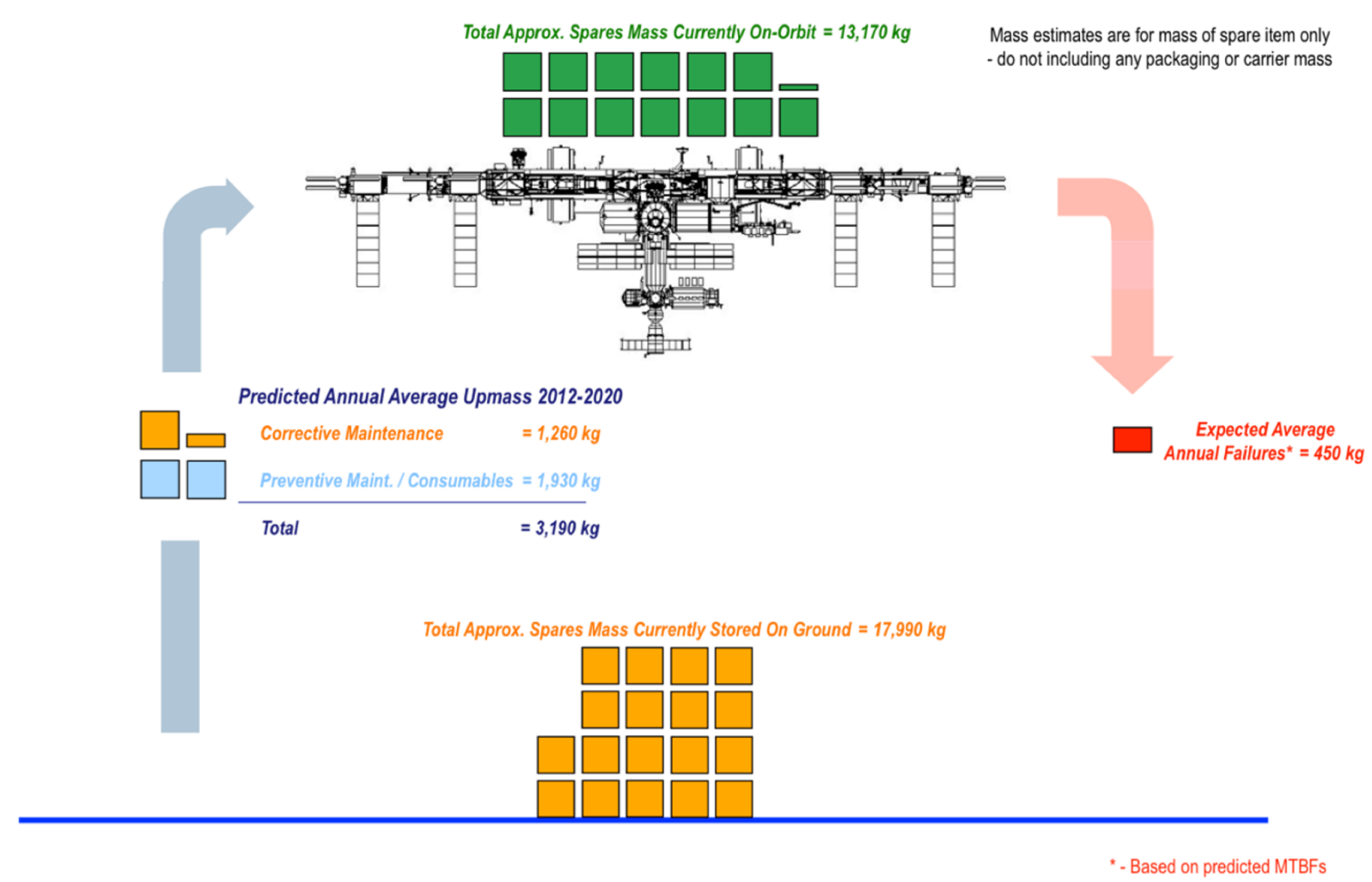

Figure 1. Estimated ISS maintenance logistics for 2012-2020, including ground storage, upmass, on-orbit storage, and expected utilization. Over $13,000 \mathrm{~kg}$ of spares on-orbit, along with nearly $18,000 \mathrm{~kg}$ on the ground, are required to support human spaceflight operations, even in LEO. ${ }^{2}$

result of aleatoric uncertainty in item failures and maintenance demands. Failure processes are inherently stochastic - mission planners cannot predict ahead of time exactly how many times a particular item will fail, they can only calculate a distribution representing the number of failures that may be experienced. The total expected number of failures (and therefore spares mass required) may be low, but exactly which items will fail is unknown. Since a particular spare can only cover one type of failure (or, in the case of common components, some subset of possible failures), spares have to be carried that will not be used in order to drive down risk. As a result, the efficiency of maintenance logistics mass - defined as the percentage of mass carried that is actually used - is fairly low when traditional sparing approaches are used. For example, Cirillo et al. estimated that more than $95 \%$ of spares manifested for corrective maintenance would not be used. $^{2}$ In LEO, when regular resupply flights can replenish spares stockpiles when a failure occurs, risk can be covered by a small number of on-board spares that are replaced when they are used. This option will not be available on deep space missions, and therefore spares stockpiles will have to cover multiple failures of the same component, which will result in an increase in mass.

\section{Unanticipated Issues}

In addition to logistics challenges from anticipated failures, ISS experience has shown that unanticipated issues arise during the operation of complex spacecraft systems, even after years of testing and on-orbit experience. This is particularly true for the regenerative Environmental Control and Life Support (ECLS) systems, which are critical for crew survival. Do ${ }^{11}$ compiled a summary of major issues experienced by ISS regenerative ECLS, including:

- damage to Carbon Dioxide Removal Assembly (CDRA) check valves caused by dust and debris from sorbent pellets, ${ }^{13,14}$

- failure of the Urine Processor Assembly (UPA) distillation assembly caused by precipitation of calcium sulfate due to unexpectedly high concentrations of calcium in astronaut urine (a result of bone loss in the microgravity environment), forcing a reduction in water recovery rate, ${ }^{15,16}$ 
- degradation of Water Processor Assembly (WPA) catalytic reactor seals caused by prolonged exposure to reactor operating temperatures, resulting in water leakage, ${ }^{16}$ and

- multiple failures of the UPA Fluids Control and Pump Assembly (FCPA) due to manufacturing and assembly errors, including insufficient lubrication, parts misalignment during assembly, and incorrect machining resulting from misinterpretation of design drawings. ${ }^{16}$

These unanticipated failures often result from environmental interactions or manufacturing defects, and have typically been addressed in the short term with operational workarounds that accept degraded system performance. In the longer term, systems and components are redesigned (or new components are added to the system) in order to implement a more permanent fix. All of these solutions rely extensively on ground support, either to make up for degraded performance or to supply new parts, and these options will not be available on deep space missions. The fact that unanticipated issues continue to appear after years of ISS operations implies that very extensive all-up systems testing in a relevant environment will be required to try to identify as many of these issues as possible; however, it is unlikely that all eventualities can be identified and mitigated ahead of time for a multi-year mission to a new destination.

\section{Parameter Uncertainty}

The aleatoric uncertainty that is inherent to maintenance demand forecasting is compounded by epistemic uncertainty in our knowledge of key parameters, especially component failure rates. The ISS has been and continues to be an excellent and necessary testbed that can be used to gather data on component performance and reliability in a spaceflight environment. However, the sample size available for analysis is extremely limited, with only one instance of most systems implemented on one space station, often for less than a decade. ${ }^{17}$ As a result, the statistical significance of data used for estimates of component reliability is limited, and may be based heavily on prior assumptions even with the Bayesian MTBF updating process that is implemented. ${ }^{18}$ This low sample size means that significant uncertainty remains in failure rate estimates. In addition, the operating environment of future systems will be different from LEO, especially for missions to Mars. These different environments may impact component reliability and represent an additional uncertainty that must be taken into account.

Stromgren et al. ${ }^{19}$ examined this uncertainty by looking at variation in MTBF estimates for ISS components from their initial values. While approximately $85 \%$ of components experienced an increase in reliability (higher MTBF), 15\% experienced lower-than-expected reliability. Due to the asymmetric impacts of variations in reliability, lower reliability in even a few components can significantly degrade overall system reliability, even if other components become more reliable. ${ }^{19,20}$ As a result, the general effect of this parameter uncertainty is an increase in the amount of mass required to achieve a given risk target (the relationship between mass and risk is discussed in greater detail in section $\mathrm{C}$ ). A more detailed analysis and discussion of the impacts of MTBF uncertainty is presented by Stromgren et al. ${ }^{19}$

\section{Direct Link Between Mass and Risk to Crew for Exploration Missions}

A key metric for spares manifesting is Probability of Sufficiency (POS), or the probability that enough spares are provided to cover all failures during the mission. ${ }^{21}$ For missions in LEO such as the ISS, POS is mostly decoupled from Probability of Loss of Crew $(\mathrm{P}(\mathrm{LoC}))$ because new spares can be provided on demand if supplies run short. In an extreme case, the crew always has the option to abort and return to Earth. For deep space missions, however, these options will not be available. Insufficient spares could result in loss of function in a critical system, which would then lead to loss of crew since the timescales for resupply or abort are much longer than the timescales of failure impacts. As a result, the crew's probability of survival is directly related to the amount of spare parts and other maintenance equipment they have available to them, as well as redundancy and contingency supplies. Mathematically, POS for critical systems provides a bound on $\mathrm{P}(\mathrm{LoC}):^{22}$

$$
P(L o C) \geq 1-P O S
$$

This is because system failure due to insufficient spares is only one of many situations that could lead to loss of crew, and is therefore only one of many contributors to this risk. In this paper, we refer to this bound 
(1 - POS) as the maintenance logistics contribution to $\mathrm{P}(\mathrm{LoC})$. When other, non-maintenance sources of risk are included (e.g. launch, radiation, Entry, Descent, and Landing (EDL), and other general medical risks), $\mathrm{P}(\mathrm{LoC})$ will increase. This bounding has significant implications for maintenance logistics planning for missions without abort or resupply, since the amount (and therefore mass) of spares and contingency supplies provided sets a limit on the minimum risk that can be achieved for that mission. In addition, as shown in 3 , the cost of decreasing risk is exponential growth in maintenance logistics mass.

\section{Challenges for Future Missions}

The heavily Earth-dependent maintenance logistics support strategy utilized by the ISS will not be effective for future deep space missions. Logistics requirements will increase as mission endurances get longer, and the implementation of new systems to reduce consumables logistics (especially for ECLS) typically incurs additional maintenance logistics demand, under some conditions to the point where the additional mass of the additional system and spare parts outweighs the mass of consumables saved by those new systems. For long endurance missions, spare parts can account for more than half of the total logistics mass for ECLS systems alone. ${ }^{22}$ The concept of increasing reliability to reduce logistics mass is likely not an effective solution, since it has marginal returns and incurs exponential cost and significant amounts of testing time. ${ }^{20}$ In addition, uncertainty will increase as planning horizons are extended to multi-year missions, and as systems begin operating in environments that are not as well understood. An unexpected anomaly that can be adapted to in LEO may be a critical failure for a Mars mission, where abort and resupply are not an option. Degraded system operation may result in insufficient consumables for the crew. If a new failure mode appears that reduces the reliability and lifetime of all components of a particular type (such as dust damage to CDRA valves), the spares that were provided at the start of the mission may no longer be sufficient to cover all failures. Without the ability to adapt to unexpected circumstances, the risk reduction value of traditional spare parts can be significantly reduced by common cause failures. ${ }^{23}$

Given these challenges, changes to the current approach to system maintainability need to be made in order to lower cost and risk and enable future missions. Specifically, new systems and maintenance paradigms need to be developed that:

- reduce maintenance logistics required to cover risks,

- provide risk coverage that is robust to parameter uncertainty and environmental impacts on reliability during operations, and

- enable resilient systems that can adapt and mitigate risks associated with unanticipated circumstances.

ISM is one option that has the potential to address these challenges.

\section{Potential Benefits of In-Space Manufacturing}

This section presents a brief overview of potential applications of ISM that have been proposed and a discussion of how they might impact spaceflight logistics and operations. This is not meant to be an exhaustive review, simply a set of example cases focused on applications related to Intravehicular Maintenance (IVA) maintenance. A more extensive review focusing on applications of AM for spaceflight was published by the National Research Council (NRC) in $2014 .{ }^{6}$ While AM is a very attractive technology to implement as an ISM capability, and current implementations of ISM on the ISS are AM-based systems - namely, the 3DP technology demonstration and the commercial Additive Manufacturing Facility (AMF) - it is important to keep in mind that ISM and AM are two different things. ISM is the manufacturing of items in space, via whatever means are available; AM is more specifically the production of items using additive processes. ISM can be AM, but does not necessarily have to be. Similarly, AM can provide value to spaceflight efforts whether it is implemented in space or not.

\section{A. Logistics Reduction}

One of the major benefits of an ISM capability is the fact that it allows on-demand manufacturing of spares, which means that raw materials are only specialized into specific components when needed. This "just-in-time" manufacturing capability provides a flexibility in maintenance logistics planning that helps 
reduce uncertainty, thereby reducing the number of items that need to be held in a spares inventory. Whereas a spare part can only cover a single type of failure (or a subset of failures, if commonality is implemented), a manufacturing capability would be able to cover several different types of failures using the same undifferentiated mass. ${ }^{9,24-26}$ Put another way, ISM enables commonality of material rather than commonality of design - raw materials can be converted into specific components in response to specific failures, enabling the mass reduction benefits of design while avoiding the need to enforce common design for different applications. As a result, the risk reduction per unit mass of raw materials is higher than for discrete spares, since those raw materials provide a broad risk coverage. ${ }^{9}$

ISM can also simplify inventory management, both in terms of operational complexity and in terms of stowage volume and mass overhead. With an ISM capability, the number of different types of spares that need to be managed could be reduced; instead, the inventory management system would track aggregate stores of raw materials. ${ }^{27}$ In addition, raw materials tend to be much more volumetrically compact than spare parts, reducing stowage volume requirements. In 2014, the NRC estimated that raw material for AM could be packaged for launch at densities 100 times greater than current capabilities. ${ }^{6}$ Raw materials are also likely to be much more robust to launch loads than completed spares, which would further reduce packaging overhead by reducing the need for launch supports or foam. ${ }^{25}$ In addition to increasing launch volumetric efficiency, this increased density could also enable increased habitable volume for the crew or smaller spacecraft overall for future missions. ${ }^{6,7,27,28}$

As noted in section II, one of the traditional approaches to logistics reduction for consumables related to ECLS or propulsion is recycling or ISRU. While these approaches can be extremely effective at reducing logistics requirements related to chemicals, recycling or ISRU cannot be applied to maintenance logistics unless a manufacturing capability is also available since the end goal is a physical component with a specific geometry. With ISM, however, these options - and the associated logistics reduction benefits - become available. Materials recycling could "close the loop" on spare parts and allow the same raw material to be used multiple times, significantly reducing logistics requirements. ${ }^{6,25,29-31}$ This materials recycling could be applied to re-manufacture failed components into new components, or it could enable system reconfiguration, where components are recycled into different elements as the mission moves through different phases in order to adapt to current conditions. Materials recycling could also take advantage of obsolete or defunct spacecraft from previous missions, since they likely represent the highest concentration of preprocessed aerospacegrade material that will be found outside the atmosphere. ${ }^{30}$ Scavenging materials and components from a predeployment system could enable the raw materials of a Mars lander or a spent in-space propulsion stage to be part of the logistics for a mission to Mars. This type of materials scavenging may in practice be similar to the Defense Advanced Research Projects Agency (DARPA) Phoenix program, which is looking at repurposing valuable components from retired satellite systems. ${ }^{6}$ Materials recycling also provides a means to reduce waste stowage and disposal requirements, which may be valuable from a planetary protection perspective for surface missions. ${ }^{25}$ ISRU for manufacturing has the potential to eliminate, rather than reduce, the need to launch some materials from Earth in the first place. This could result in very significant mass reductions, especially for long missions with heavily-constrained resupply options. ${ }^{6,7,24,30}$

\section{B. Adaptability}

ISM also provides the capability to adapt to changing circumstances. Rather than being constrained to the set of spares that were provided at the start of the mission, the crew could use ISM to create a new item (or adjust the design of an existing one), potentially defeating common cause failures and allowing the system to evolve and adjust to unanticipated conditions. In addition, ISM allows rebalancing of maintenance logistics resources (i.e. raw materials) in response to variation in component failure rates; mass that was initially allocated for a spare that experiences better-than-planned reliability can be reallocated to cover failures of a spare that experiences worse-than-planned reliability.

Replacement components manufactured via ISM could be full, nominal replacements, with equivalent or near-equivalent properties to the original component. However, they could also be contingency options, or temporary solutions that allow the system to continue functioning in a degraded state until a more permanent solution can be developed. ${ }^{7,27,32}$ This palliative approach to maintenance mimics biological responses to damage, allowing the system to first mitigate the impacts of a failure with a temporary fix (i.e. a part with lower quality than a standard part) before implementing a longer-term solution. ${ }^{32}$ A temporary fix may relax the requirements placed on an ISM system, ${ }^{7}$ making ISM easier to implement in the near term and enabling utilization while more advanced manufacturing capabilities are being developed. For example, it 
may be that a plastic part manufactured via fused filament deposition - a process that has already been demonstrated on orbit by 3DP - could be used as a temporary solution to an emerging issue on the ISS while the crew waits for a more permanent solution to be delivered from the ground. This palliative capability would allow the system to adapt and mitigate the impacts of failures in the short term, potentially reducing system downtime and allowing the resumption of operations without requiring a resupply or time-consuming operational workarounds.

\section{Novel Capabilities}

ISM is a means to remove constraints from space systems design, particularly those related to launch. A system manufactured in space does not have to survive launch loads (acceleration or vibration), nor does it have to fit within a launch fairing. If AM is used, many geometry constraints associated with manufacturing limitations may also be removed. This new design freedom enables re-optimization of system design that may significantly reduce mass or enable entirely new capabilities. ${ }^{6,7,25,27,28}$

AM has enabled significant new manufacturing capabilities on the ground and could provide similar benefits in space. For example, re-optimization of a structural element to take advantage of the design flexibility provided by AM resulted in an element that could support the same structural loads while using $75 \%$ less mass. ${ }^{33} \mathrm{AM}$ can also enable parts consolidation, where parts that due to manufacturing constraints would previously need to be bolted or welded together can now be manufactured as a single piece. For example, recent efforts to produce a rocket fuel pump using AM have enabled a $45 \%$ reduction in the number of parts required. ${ }^{34}$ A reduction in parts count can help simplify assembly and maintenance processes and reduce the number of attachment points within a system, which could reduce system complexity and save crew time. ${ }^{26,30,35}$ A combination of these two capabilities was recently demonstrated when a spacecraft bracket was re-optimized for AM. The removal of manufacturing constraints allowed the original part which had required 4 pieces and 44 rivets - to be redesigned into a single part, resulting in a $35 \%$ reduction in mass as well as a $40 \%$ increase in stiffness. ${ }^{36}$

A similar optimization could be applied to spacecraft structures or components in space, with the only forces included being the forces it will experience during operations. A system that will spend its entire operating lifetime in a microgravity environment does not need to be able to withstand launch loads if it can be manufactured in that environment. This capability could, for example, enable hyper-efficient structural design to create low inert mass fraction spacecraft or in-space propulsion systems, which could produce compounding benefits from reduction in propellant mass requirements.

\section{Quantitative Assessment of Logistics and Risk Impacts}

To quantify the impact of ISM on maintenance logistics mass requirements, this paper examines the impact of ISM on the total logistics mass required to achieve a given risk target. The mission profile analyzed here examines the EMC DSH on a nominal 1,100 day mission to Mars and back, calculating spare parts requirements for critical systems - including ECLS (atmosphere control, thermal control, and water recovery and management), attitude and rate determination, command and data handling, communications and tracking, and power - for both corrective maintenance (i.e. random failures) and preventative maintenance (i.e. scheduled repair). The three cases examined are:

- Traditional Spares: spare parts manufactured on Earth are manifested, and no ISM capability is available.

- ISM: a notional ISM capability is provided that uses raw materials brought from Earth. For the purposes of this paper, the ISM capability covers primarily items related to fluid flow, including plumbing, ducting, fans, heat exchangers, tanks, and valves (in total, approximately $33 \%$ of line items in the Master Equipment List (MEL)).

- ISM + Recycling: the ISM capability described above is augmented with the capability to recycle raw materials. It is assumed that material from failed spares can be recycled back into usable feedstock up to 10 times.

The notional ISM and recycling capabilities represented in these cases are meant as examples only, and do not represent a claim that the ability to manufacture these items currently exists. The purpose of the analyses 
presented here is to examine the value that such a capability could provide if it could be developed. For each case, the total mass of spares and ISM feedstock required is calculated, along with packaging overhead. Note that the mass of emplaced systems are not included, nor is the mass of the ISM/recycling system or the spares required to maintain them. These results indicate the change in maintenance logistics mass when ISM and/or feedstock recycling are available, and this reduction in logistics mass can be thought of as the mass budget to implement these systems.

A range of POS values between 0.9 and 0.999999 is examined, corresponding to a maintenance logistics contribution to $\mathrm{P}(\mathrm{LoC})$ ranging from $10^{-1}$ to $10^{-6}$. For each risk level, a spares manifest is first calculated using deterministic MTBF estimates, then a Monte Carlo simulation is run incorporating uncertainty in MTBF values in order to determine the median risk actually covered by that manifest, along with $25^{\text {th }}$ and $75^{\text {th }}$ percentile values. The result is a set of curves indicating the mass required to achieve a given level of maintenance logistics contribution to $\mathrm{P}(\mathrm{LoC})$, either under the assumption that MTBF values are fixed (i.e. the deterministic result) or to achieve a given $25^{\text {th }} / 50^{\text {th }} / 75^{\text {th }}$ percentile contribution to $\mathrm{P}(\mathrm{LoC})$ when MTBF uncertainty is taken into account.

In addition, the impact of MTBF uncertainty is examined in greater detail for a specific baseline, where the maintenance logistics contribution to $\mathrm{P}(\mathrm{LoC})$ is $10^{-3}$. The Monte Carlo simulation described above is applied to the manifest that achieves that risk threshold when MTBFs are assumed to be deterministically known in order to determine the distribution of contribution to $\mathrm{P}(\mathrm{LoC})$ actually achieved when uncertainty in MTBF values is taken into account. Of particular interest are the median contribution to $\mathrm{P}(\mathrm{LoC})$ after uncertainty, as well as the probability of achieving the original target value when uncertainty is taken into account. This analysis is run for the traditional spares case and the ISM case (i.e. $33 \%$ of items considered manufacturable). In addition, a notional case was created by arbitrarily setting half of the components to be manufacturable in order to examine how these impacts trend with changes in ISM capability.

\section{A. Model Description}

\section{Maintenance Demand and Manifest Optimization}

For this analysis, component failures are modeled using a Poisson distribution in order to determine demand for spare parts. It is assumed that all component failures are independent, all repairs are completed successfully, and that the impact of system downtime while repairs are being implemented are negligible. The Cumulative Distribution Function (CDF) for the Poisson distribution then represents the number of spares that will be required for a given item, and can be used to calculate the individual POS for that item, $P O S_{i}$, as a function of the number of spares carried for that item. The overall POS is the product of the POS for each individual item. The manifest optimizer then applies a branch-and-bound discrete optimization algorithm $^{21,37}$ to determine the combination of spares that achieves a target POS value for minimum mass. The maintenance logistics contribution to $\mathrm{P}(\mathrm{LoC})$ is equal to 1 minus the overall POS achieved. Scheduled maintenance demands are accounted for in the manifest optimization algorithm by setting a lower bound on the number of spares required for life-limited items. A more detailed description of the maintenance demand and manifest optimization approach is presented by Owens and de Weck. ${ }^{22}$

The above procedure determines the number of spares required for each item when a traditional sparing approach is used. When ISM is available, some of these spares are covered from a common pool of raw material, or feedstock - this is the commonality of material effect described in section III. In this case, traditional spares for manufacturable items are removed from the manifest and the mass of feedstock required to cover their potential failures to the same POS level is calculated using the procedure shown in figure $2 .{ }^{9}$ For each manufacturable item, the Probability Mass Function (PMF) of the number of corrective maintenance spares required (i.e. the number of spares beyond those provided for deterministic scheduled maintenance) is first stretched by the mass of that spare in order to generate a PMF representing the total mass of that spare that will be required. These spares mass PMFs are then convolved together in order to calculate the PMF for the total mass of spares that will be required for manufacturable items. This distribution is then used to select the mass of feedstock required to achieve POS targets. Note that this procedure assumes that all spares covered by ISM can be manufactured from the same raw material; however, distributions for multiple different types of feedstock can also be generated given sufficiently detailed knowledge of spares material composition. A more detailed description of this feedstock modeling approach is presented by Owens et al. ${ }^{9}$

The result of the above procedure is a total feedstock requirement; when feedstock recycling is available, this amount is divided by the number of times that a particular unit of feedstock can be recycled. However, 

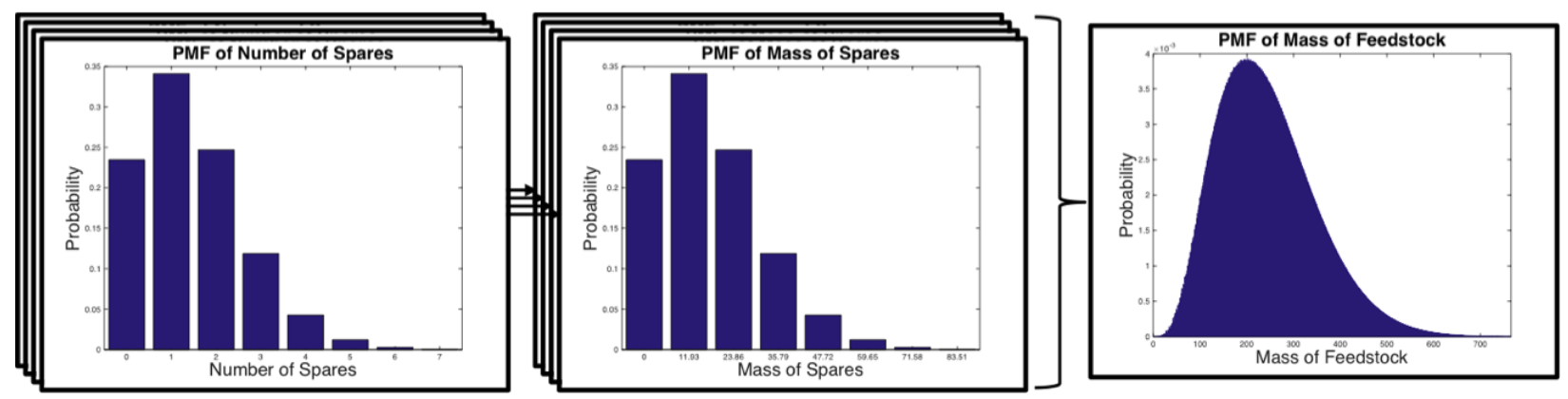

Figure 2. Procedure used to determine the mass of feedstock required to cover failures of items that can be manufactured via ISM. ${ }^{9}$

the minimum mass of feedstock is limited to the mass of the largest spare to be covered by ISM in order to ensure that a new spare can be printed on demand without having to wait to recycle a failed item. This constraint may be revisited in future investigations, depending on the time to critical impact after failure and the risk posture of a given mission. For the purposes of this analysis, it is assumed that a given unit of feedstock can be recycled up to 10 times.

Once the number of spares provided for each item and the total amount of feedstock required are determined, the total maintenance logistics mass is calculated. Spares counts are multiplied by the mass of the corresponding spare and summed to determine the total spares mass. In addition, a packaging overhead of $1.5 \%$ by mass is applied to spare parts to account for foam and other packaging requirements for spares. ${ }^{38}$ It is assumed that raw material can achieve 100 times greater volumetric density than traditional spares, ${ }^{6}$ and therefore the packaging overhead applied to feedstock is $0.015 \%$. This reduction in packaging overhead for feedstock also accounts for the fact that raw materials will likely be much more robust to launch loads than finished spares and will therefore require less foam and packaging materials for protection. ${ }^{25}$ The total maintenance logistics mass is the sum of the mass of spares, the mass of feedstock, and the mass of packaging material.

\section{Accounting for MTBF Uncertainty}

The procedure described in section 1 calculates the total maintenance logistics mass required to achieve a given risk level under the assumption that all component MTBF values are known. However, significant epistemic uncertainty in MTBF values may remain that will decrease POS and increase risk for a given set of spares. The net result is that a significantly higher mass is required to achieve a given risk target, as described in section II. ${ }^{19}$ In order to account for the impact of MTBF uncertainty, a Monte Carlo simulation was executed in which 75,000 simulations were run with a given manifest. In each simulation, a new failure rate for each component was selected according to the distribution of MTBF changes observed on the ISS. More details on the distribution used are presented by Stromgren et al. $;^{19}$ approximately $85 \%$ of items experienced an increase in reliability, and approximately $15 \%$ experienced a decrease. ${ }^{19}$ Once MTBFs are updated according to this random selection, the overall maintenance logistics contribution to $\mathrm{P}(\mathrm{LoC})$ was recalculated for that manifest for each run. Statistical analysis of the results of the Monte Carlo simulation were then used to determine the median and $25^{\text {th }} / 75^{\text {th }}$ percentile values for contribution to $\mathrm{P}(\mathrm{LoC})$ actually achieved by manifest. Since the contribution to $\mathrm{P}(\mathrm{LoC})$ is higher when uncertainty is included, the mean and quartile results do not cover the entire range of risk values examined in the deterministic analysis. Therefore, a curve fit is used to extrapolate these results across the remaining range of maintenance logistics contribution to $\mathrm{P}(\mathrm{LoC})$.

\section{B. Results}

Figure 3 shows the logistics mass required to achieve a desired risk value for the traditional case and cases with ISM and ISM with material recycling. The manifest required to provide a median a contribution to $\mathrm{P}(\mathrm{LoC})$ of $10^{-3}$ has a mass of $17,232 \mathrm{~kg}$, and approximately $4,124 \mathrm{~kg}$ must be added for each additional order of magnitude reduction in contribution to $\mathrm{P}(\mathrm{LoC})$. When ISM is available, the mass required at this risk level is $12,323 \mathrm{~kg}$, a reduction of approximately $28 \%$. The mass cost of additional risk coverage is also reduced, with each additional order of magnitude reduction in contribution to $\mathrm{P}(\mathrm{LoC})$ requiring an additional $2,900 \mathrm{~kg}$, 


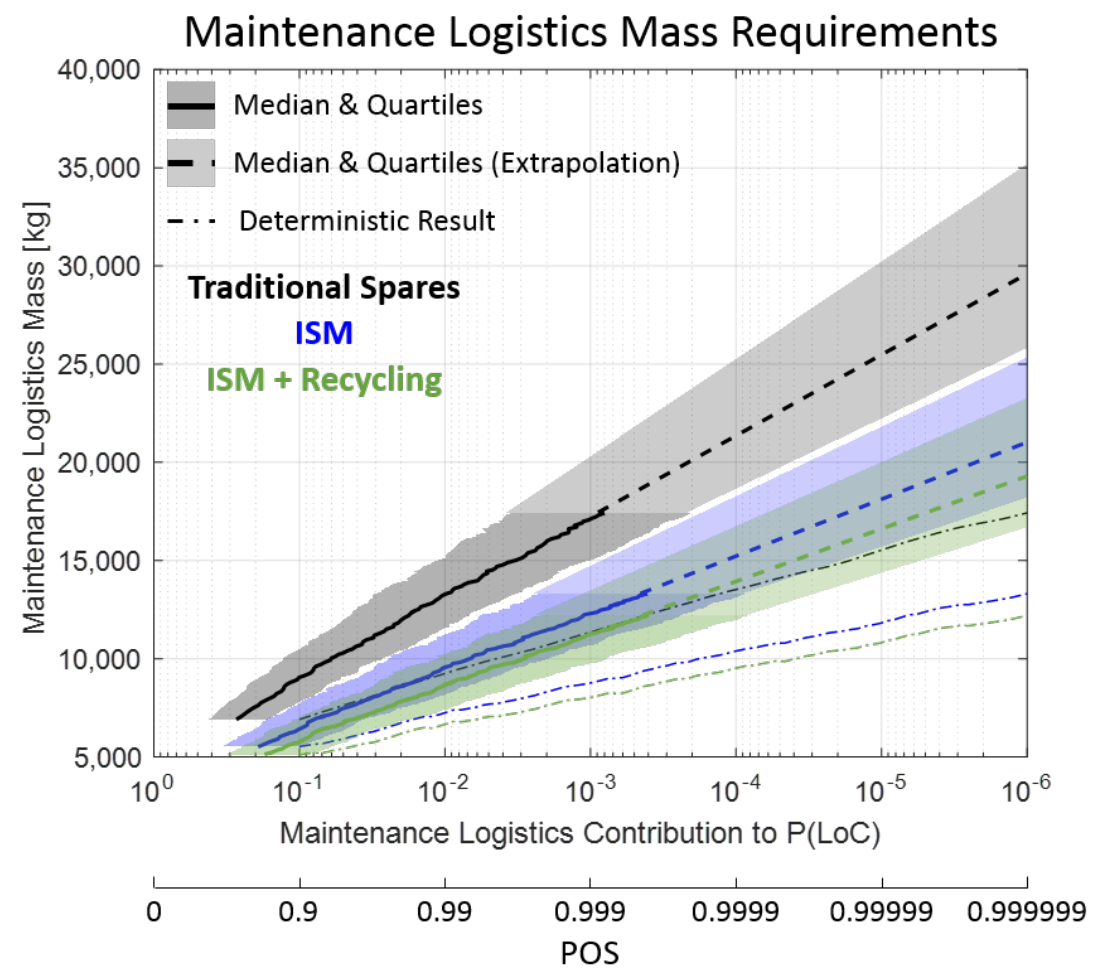

Figure 3. Maintenance logistics mass required for a 1,100 day DSH mission as a function of contribution to P(LoC) for traditional spares (black), ISM (blue), and ISM with recycling (green). Median and $25^{\text {th }} / 75^{\text {th }}$ percentile values are shown by the line and shaded region, and the deterministic result (without MTBF uncertainty) is shown by the thin dotted line.

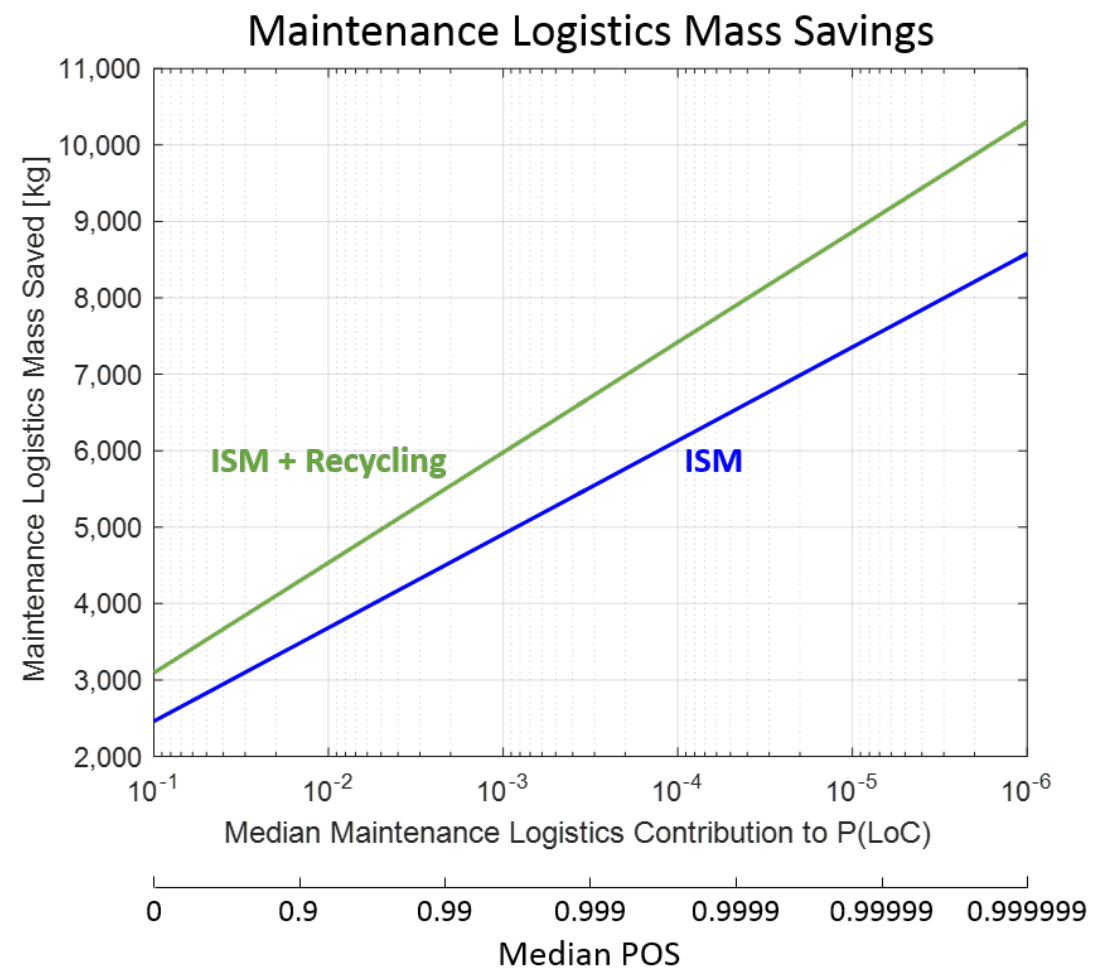

Figure 4. Reduction in DSH maintenance logistics mass from traditional case achieved when ISM (blue) or ISM and material recycling (green) are used (based on median risk after MTBF uncertainty is included). These results indicate the change in maintenance logistics mass for DSH critical systems examined here, and do not include the mass of the ISM system itself or any supporting infrastructure and maintenance mass requirements. 
a $30 \%$ reduction of the mass cost to decrease risk compared to traditional spares. When a material recycling capability is added, the mass required at a POS of 0.999 is $11,254 \mathrm{~kg}$ - an approximately $34 \%$ reduction from traditional spares, and an $8.7 \%$ reduction from the ISM case - and the mass growth per order of magnitude reduction in contribution to $\mathrm{P}(\mathrm{LoC})$ is reduced further to $2,681 \mathrm{~kg}$, or $35 \%$ less than the traditional spares case.

Figure 4 shows the total maintenance logistics mass saved when ISM and material recycling are added as a function of the median contribution to $\mathrm{P}(\mathrm{LoC})$ achieved, based on curve fits to the median results shown in figure 3. The commonality of material and reduced packaging overhead enabled by ISM saves $4,909 \mathrm{~kg}$ of maintenance logistics mass required to achieve a baseline median contribution to $\mathrm{P}(\mathrm{LoC})$ of $10^{-3}$. In addition, since the rate of mass growth with risk reduction is lower when ISM is available, the maintenance logistics mass saved increases as maintenance contribution to $\mathrm{P}(\mathrm{LoC})$ is reduced, with each order of magnitude increase in risk reduction targets corresponding to an increase in mass savings of $1,204 \mathrm{~kg}$. When feedstock recycling is available, the maintenance logistics mass saved at the baseline risk level is $5,978 \mathrm{~kg}$, and each order of magnitude reduction in risk corresponds to additional $1,443 \mathrm{~kg}$ of mass saved from the baseline. Note that these numbers do not include the mass of the ISM system itself, or its infrastructure and maintenance mass requirements; they only indicate the impact of ISM on the maintenance logistics mass required for DSH critical systems.

Figure 5 examines the "mass compression" achieved by ISM and material recycling - that is, the difference between the mass that was originally required for the components that were manufactured via ISM and the mass of feedstock required to cover them when ISM is available. For the set of components examined in this notional case - approximately a third of items in the MEL - the maintenance logistics mass required (including packaging overhead) is $5,318 \mathrm{~kg}$ at the baseline target contribution to $\mathrm{P}(\mathrm{LoC})$ of $10^{-3}$, or approximately $31 \%$ of the total maintenance logistics mass required for the traditional spares case at this risk level. When ISM is available, these same items are covered by $1,156 \mathrm{~kg}$ of feedstock (including packaging). This represents a $78.3 \%$ reduction in maintenance logistics mass requirements for these items. Feedstock recycling reduces the mass even further: only $125 \mathrm{~kg}$ of feedstock and packaging are required to achieve the same risk coverage for these items - a $97.7 \%$ reduction from the mass required for traditional spares - when both ISM and feedstock recycling are available.

Figure 6 shows the impact of MTBF uncertainty on the contribution to $\mathrm{P}(\mathrm{LoC})$ after uncertainty is incorporated. The manifest examined here was created using current MTBF estimates to achieve a target maintenance logistics contribution to $\mathrm{P}(\mathrm{LoC})$ of $10^{-3}$, then a Monte Carlo analysis of this manifest was implemented using the procedure described above, where individual item MTBFs were varied according to the uncertainty distribution described by Stromgren et al. ${ }^{19}$ Figure 6 clearly demonstrates the asymmetric impact of variations in MTBF. Even though each item had an $85 \%$ chance of experiencing higher reliability, the net effect of uncertainty tended to increase contribution to $\mathrm{P}(\mathrm{LoC})$. However, ISM can reduce the impact of this effect, since it enables redistribution of maintenance logistics resources in response to variation in MTBFs.

When traditional spares are used, the median contribution to $\mathrm{P}(\mathrm{LoC})$ increases to 0.0270 , and the probability that the contribution to $\mathrm{P}(\mathrm{LoC})$ will be higher than the target value of $10^{-3}$ is 0.9948 ; in almost all cases, overall system reliability is lowered when MTBF uncertainty is accounted for. When $33 \%$ of items can be manufactured via ISM (the same ISM case used in the mass reduction results above), the median contribution to $\mathrm{P}(\mathrm{LoC})$ after uncertainty is reduced to 0.0167 . The probability that the contribution to $\mathrm{P}(\mathrm{LoC})$ will be increase when uncertainty is accounted for is also reduced, to a value of 0.9633 . When ISM capability is increased to have the ability to manufacture $50 \%$ of items, the median contribution to $\mathrm{P}(\mathrm{LoC})$

Table 1. Summary of the impact of ISM capability on the effects of MTBF uncertainty when applied to a manifest optimized to achieve a maintenance logistics contribution to $P(L o C)$ of $10^{-3}$ in the deterministic case. The median maintenance logistics contribution to $\mathrm{P}(\mathrm{LoC})$ and the probability that the net effect of uncertainty in MTBF values will be an increase in contribution to $\mathrm{P}(\mathrm{LoC})$ are tabulated for cases where traditional spares are used and where $33 \%$ or $50 \%$ of items can be manufactured via ISM.

\begin{tabular}{ccc}
\hline & $\begin{array}{c}\text { Median Contribution } \\
\text { to P(LoC) }\end{array}$ & $\begin{array}{c}\text { Probability of Increased } \\
\text { Contribution to P(LoC) }\end{array}$ \\
\hline Traditional Spares & 0.0270 & 0.9948 \\
ISM (33\%) & 0.0167 & 0.9633 \\
ISM (50\%) & 0.0084 & 0.8981 \\
\hline
\end{tabular}




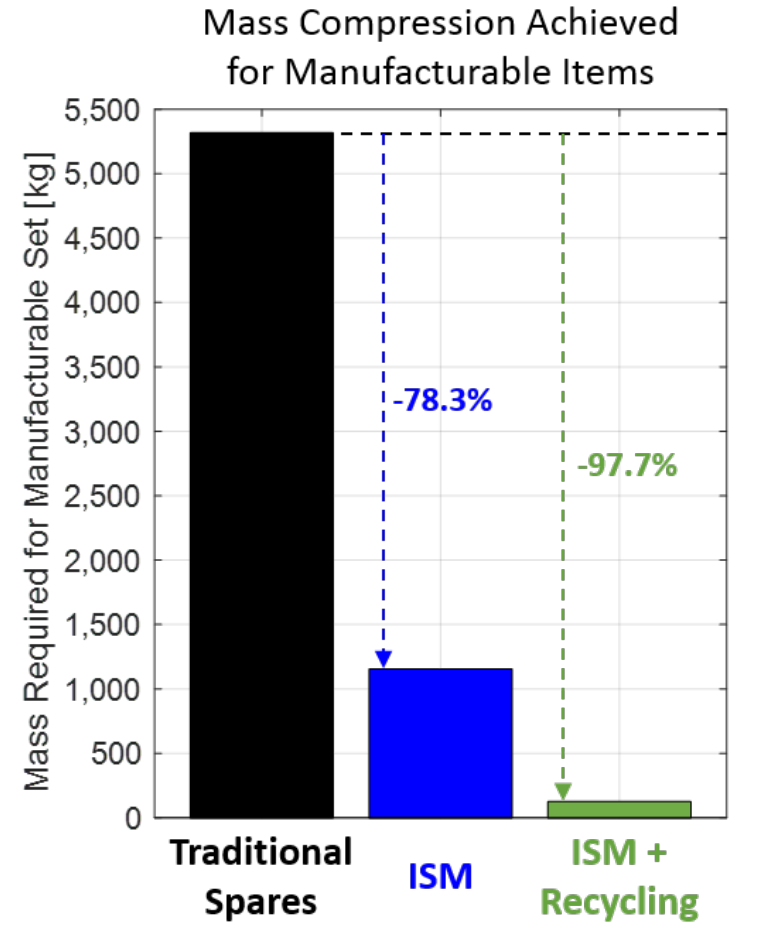

Figure 5. Mass compression achieved for items that ISM was applied to, based on the manifest required to achieve a median contribution to $\mathrm{P}(\mathrm{LoC})$ of $10^{-3}$. The maintenance logistics mass associated with spares that are assumed to be manufacturable via ISM in this analysis is shown when they are covered by traditional spares (black), ISM (blue), and ISM with recycling (green).

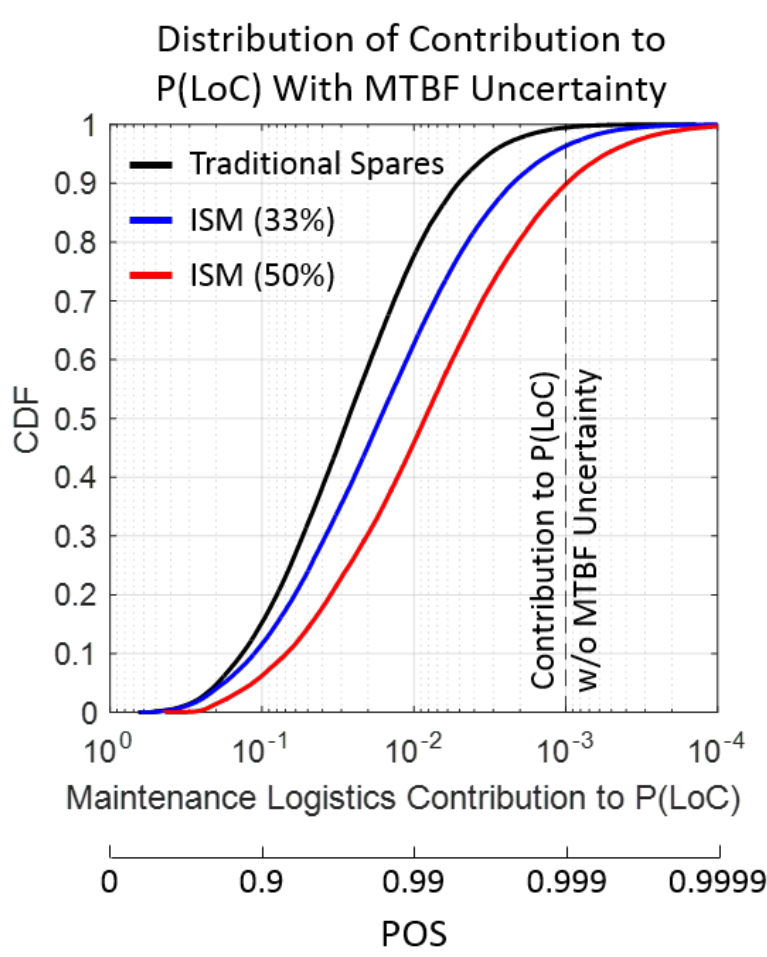

Figure 6. Distribution of maintenance logistics contribution to $\mathrm{P}(\mathrm{LoC})$ when MTBF uncertainty is incorporated. The target value for manifesting using current MTBF estimates was $10^{-3}$. Results are compared for the case using traditional spares (black) and the ISM capability examined in this case study (33\% of items), as well as a notional case with increased ISM capability ( $50 \%$ of items).

is further reduced to 0.0084 , or approximately $31 \%$ of the value for traditional spares. The probability that the contribution to $\mathrm{P}(\mathrm{LoC})$ will increase is similarly reduced to 0.8981. These results are summarized in table 1.

\section{Discussion}

\section{A. Reduction of Maintenance Logistics}

The above results indicate that ISM has the potential to significantly decrease maintenance logistics mass for future exploration systems. Mass and risk are tightly coupled for deep space missions, and figure 3 shows that when traditional spares-based approaches are used to maintain systems and drive down risk, logistics mass grows very quickly. Uncertainty is a major challenge for maintenance logistics planning, both in terms of the aleatoric uncertainty present in component failure and repair processes and in terms of the epistemic uncertainty in our understanding of these processes. ${ }^{2,19}$ As a result, when the current approach to maintenance logistics is applied to long-endurance missions beyond LEO large amounts of mass must be carried to drive down risk. Importantly, as noted previously, the risk of not having enough maintenance supplies is only one of many risks to the crew, so the maintenance logistics contribution to $\mathrm{P}(\mathrm{LoC})$ indicated on the $\mathrm{x}$-axis of figures 3 and 4 are bounds on the level of crew safety that can be achieved on a given mission. These charts therefore indicate the mass required to be able to achieve a desired level of safety, not the mass that actually achieves that level. For example, in the Mars transit DSH case examined here 17,232kg are required to drive the $\mathrm{P}(\mathrm{LoC})$ due to maintenance logistics down to a median value of 1 in 1000, and more mass would need to be carried if the overall mission $\mathrm{P}(\mathrm{LoC})$ target is 1 in 1000 , since the actual risk to the crew will be higher than the risk indicated in these charts when all other sources of risk are considered.

ISM helps mitigate some of the detrimental effects of both aleatoric and epistemic uncertainty by providing additional flexibility in the maintenance logistics process. Raw materials provided for ISM do not need to 
be specialized to a specific spare part until a demand is known ("just-in-time" manufacturing). As a result, a given unit of raw mass can cover a broader range of failure modes than a specific spare, and maintenance logistics can be reduced due to greater risk coverage efficiency. ${ }^{9}$ Effectively, this commonality of material reduces the impact of aleatoric uncertainty and enables significant reduction in maintenance logistics mass required to achieve a given risk level - continuing the example from above (contribution to $\mathrm{P}(\mathrm{LoC})$ of 1 in 1000), ISM allows a $28 \%$ reduction in maintenance logistics mass, reducing the mass required to achieve the same level of risk to $12,323 \mathrm{~kg}$.

In addition to the benefits provided by commonality of material, ISM opens the possibility of material recycling and/or ISRU to reduce maintenance logistics. This is a technique that has been used to great effect to reduce ECLS and propellant logistics requirements, but it cannot be similarly applied to maintenance logistics without an ISM capability. In the case examined above at the example risk level described in the previous paragraphs, material recycling allows a reduction of $34 \%$ from the traditional spares case, reducing the maintenance logistics mass requirement to $11,254 \mathrm{~kg}$. As the ISM capability expands to enable application to more and more components, the mass reduction impacts of both commonality of material and recycling will increase.

\section{B. Attenuation of the Impact of MTBF Uncertainty}

ISM also helps mitigate the impacts of epistemic uncertainty in item MTBFs, again by enabling flexibility. When traditional spares are used, maintenance logistics resources must be allocated ahead of time to specific failure modes. As a result, if a single type of spare exhibits reliability that is lower than anticipated, the number of spares provided for that item may no longer be sufficient and the spares provided for other failure modes will not be able to help. Due to the asymmetric impact of variation in reliability on POS, even if the majority of spares exhibit higher than expected reliability, the spares that have lower than expected reliability will significantly increase risk for the mission. ${ }^{19,20}$ However, if an ISM capability is provided, maintenance logistics resources can be rebalanced in response to unexpectedly low/high failure rates for items that are covered by ISM. If the demand for spares for a given component is lower than expected because it exhibits higher reliability, it will use less raw materials, which then increases the mass of raw materials available to other components covered by ISM. Therefore, if an item has lower than expected reliability and therefore requires more spares, this mass is available for that purpose and the overall impact on mission risk can be reduced. The results shown in figure 6 and table 1 indicate that the introduction of an ISM capability reduced both the median maintenance logistics contribution to $\mathrm{P}(\mathrm{LoC})$ after uncertainty and the probability that MTBF uncertainty would result in an increase in risk. When a greater ISM capability was introduced, both metrics were decreased even further.

\section{Implications for ISS Applications}

The case examined in section IV was for a long-endurance Mars transit habitat. For shorter-endurance missions in LEO, such as the ISS, maintenance logistics impacts will not be as high, since the planning time horizon is much shorter and therefore uncertainty is reduced. In addition, ready access to on-demand resupply, extensive Earth support, and the option to abort in the event of an emergency all provide risk mitigation options that are not available for deep space missions. However, ISM applications on the ISS could still reduce logistics demand and mitigate additional risk. These applications would not necessarily reducing $\mathrm{P}(\mathrm{LoC})$, since many loss of crew scenarios are covered by abort options. However, they may allow a reduction in Probability of Loss of Mission $(\mathrm{P}(\mathrm{LoM}))$ by reducing the probability that an abort would be required. If a critical failure occurs that would result in an unsafe condition before a resupply mission could be prepared and launched, ISM can give the crew broader capability to implement a palliative repair to maintain critical system functions.

More importantly, however, the ISS is a critical microgravity testbed to develop and validate ISM technology. These development activities can include the maintenance applications described here; however, it is important to bear in mind that ISM applications are not limited to only maintenance logistics and risk reduction. ISM could also be used to support scientific activities, or it may be used to enable entirely new capabilities. The ISS provides an experimental platform to explore the new solution space available to designers with the removal of launch constraints. 


\section{Assumptions and Limitations}

It is important to note that the mass forecasts presented in figure 3 only account for anticipated failures. As noted in section II, ISS experience demonstrates that unanticipated issues appear in spaceflight operations, and planning for future missions should expect similar issues to continue to appear. These unanticipated issues will be particularly prevalent on long missions in relatively unknown environments, and could significantly increase risk to the crew. The greater flexibility provided by ISM expands the solution space available to crewmembers in response to these unanticipated events, thereby providing risk mitigation benefits in addition to the logistics reduction benefits described above.

This analysis also assumed that all spare parts were of the same mass that they are now. As discussed in section III, design optimization to take advantage of advanced manufacturing techniques such as AM could allow for direct reduction of the mass of particular items, further reducing mass. Redesign of components to accommodate ISM capabilities may also be explored even if they result in an increase in component mass, since the logistics mass reduction benefits outweigh the mass increase resulting from less mass-efficient component design. Systems analyses such as the one presented in section IV will need to be carried out to consider the holistic impacts of changes in item mass.

In addition, these results are only for the mass of spares and packaging associated with critical DSH systems. Other systems would also require spare parts, resulting in an increase in mass. In addition, all of this logistics mass would have to be stored in a vehicle for transportation, which may incur additional infrastructure costs. Finally, all of this mass - spares, packaging, and vehicle infrastructure - will require propellant mass for transportation. Importantly, since these are spare parts to support critical habitat functions, at least some portion of this mass must travel with the crew to Mars, on trajectories that tend to be faster and less efficient. In addition, at least some portion of this mass must also travel back to Earth when the crew leaves Mars, meaning that propellant is required for the inbound trip as well as the outbound one. The propellant costs associated with this logistics mass depend on the particular transportation architecture used, and the costs and benefits of the maintenance support and transportation strategy will need to be carefully examined for proposed mission scenarios.

The modeling approach used here assumed that one stock of feedstock could be used to manufacture any items included in the manufacturable set, which is an optimistic assumption. As discussed in section IV, the modeling approach presented in figure 2 could easily be adapted to account for multiple types of feedstock, given sufficient data on the materials composition of the items to be manufactured. When different types of material are considered, some of the benefits of ISM described here will likely decrease.

The above results looked only at the impact of ISM on risk and logistics mass for existing systems. The ISM system itself will have mass that must be considered, and it will introduce new logistics demands and new risks into the system. For example, if risk coverage for maintenance relies on a manufacturing capability, and the machinery that enables that capability fails, then a broad area of risk could be left uncovered. This highlights the need for testing in a relevant spaceflight environment (i.e. the ISS) to understand the mass, logistics, and risk characteristics of ISM technology. Trade studies examining ISM applications should be continuously reevaluated as technology capabilities develop and system characteristics become better understood.

Finally, this paper makes no claims as to what types of components can be produced in space, either currently or in the future. This analysis approach instead examines what impact an ISM capability could have on maintenance logistics mass if it were implemented. The modeling framework described in section IV can be applied to examine a wide range of manufacturing capabilities, and can be updated to account more detailed aspects such as changes in part mass or reliability as a result of redesign for ISM.

\section{Conclusion}

Maintenance logistics are a major driver of risk and mass for deep space missions, and traditional logistics support techniques are heavily Earth-dependent. New strategies must be developed to enable more distant human spaceflight by reducing maintenance logistics requirements and mitigating risks via adaptable, resilient systems that are robust to uncertainty. This paper examined potential applications of ISM to address these challenges, and presented a quantitative analysis of the impact of ISM on maintenance logistics cost and risk. In summary, ISM has the potential to:

- Significantly reduce maintenance logistics mass requirements by enabling commonality of material, as 
well as opening the possibility of material recycling and ISRU for spares

- Enable robust, adaptable systems that can evolve to meet unanticipated circumstances, potentially even mitigating risks from common cause failures

- Enable novel capabilities by eliminating constraints related to launch and packaging requirements

There is likely no single "killer app" for ISM, but it is a capability with broad applications that can drive down mass and risk while simultaneously enabling new systems. Further technology development is needed to develop new processes and adapt existing advanced manufacturing techniques to the spaceflight environment, but these results indicate that if a manufacturing capability can be developed to enable on-demand, adaptable production it could have significant implications on risk and logistics for future exploration missions.

\section{Acknowledgments}

This research was supported by a NASA Space Technology Research Fellowship (NNX14AM42H) and a technical consulting contract from Jacobs Engineering in support of the NASA HEOMD AES In-Space Manufacturing Project. The authors would like to thank Bill Cirillo, Kandyce Goodliff, and Chel Stromgren for EMC DSH critical systems maintainability data and for their thoughts and input on this work. The authors would also like to thank Niki Werkheiser, Steve Newton, and everyone else from the In-Space Manufacturing team for their thoughts and input on this work.

\section{References}

${ }^{1}$ Jones, H. W., Hodgson, E. W., and Kliss, M. H., "Life Support for Deep Space and Mars," 44th International Conference on Environmental Systems, No. ICES-2014-074, Tucson, AZ, 2014, pp. 1-15.

${ }^{2}$ Cirillo, W., Aaseng, G., Goodliff, K., Stromgren, C., and Maxwell, A., "Supportability for Beyond Low Earth Orbit Missions," AIAA SPACE 2011 Conference \&S Exposition, No. AIAA-2011-7231, American Institute of Aeronautics and Astronautics, Long Beach, CA, Sep 2011, pp. 1-12.

${ }^{3}$ Hurlbert, K., Bagdigian, B., Carroll, C., Jeevarajan, A., Kliss, M., and Singh, B., "Technology Area 06: Human Health, Life Support, and Habitation Systems," Tech. Rep., National Aeronautics and Space Administration, 2012.

${ }^{4}$ Agte, J. S., Multistate Analysis and Design : Case Studies in Aerospace Design and Long Endurance Systems by, Doctoral Thesis, Massachusetts Institute of Technology, 2011.

${ }^{5}$ Agte, J., Borer, N., and de Weck, O., "Design of Long-Endurance Systems With Inherent Robustness to Partial Failures During Operations," Journal of Mechanical Design, Vol. 134, No. 10, 2012, pp. 100903.

${ }^{6}$ National Research Council, 3D Printing in Space, National Academies Press, Washington, D.C., Sep 2014.

${ }^{7}$ Johnston, M. M., Werkheiser, M. J., Snyder, M. P., and Edmunson, J. E., "3D Printing in Zero-G ISS Technology Demonstration," AIAA SPACE 2014 Conference and Exposition, No. AIAA-2014-4470, American Institute of Aeronautics and Astronautics, San Diego, CA, 2014, pp. 1-5.

${ }^{8}$ Prater, T., Bean, Q., Beshears, T., Rolin, T., Werkheiser, N., Ordonez, E., Ryan, R., and Ledbetter III, F., "Summary Report on Phase I Results from the 3D Printing in Zero-G Technology Demonstration Mission, Volume I (NASA/TP-2016219101)," 2016.

${ }^{9}$ Owens, A., Do, S., Kurtz, A., and de Weck, O., "Benefits of Additive Manufacturing for Human Exploration of Mars," 45th International Conference on Environmental Systems, No. ICES-2015-287, International Conference on Environmental Systems, Bellevue, WA, 2015.

${ }^{10}$ Kennedy, K., Alexander, L., Landis, R., Linne, D., Mclemore, C., and Santiago-Maldonado, E., "Technology Area 07: Human Exploration Destination Systems Roadmap," Tech. Rep., National Aeronautics and Space Administration, 2010.

${ }^{11}$ Do, S., Towards Earth Independence - Tradespace Exploration of Long-Duration Crewed Space System Architectures, Doctoral Thesis, Massachusetts Institute of Technology, 2016.

${ }^{12}$ Simon, M. A., Wald, S. I., Howe, A. S., and Toups, L., "Evolvable Mars Campaign Long Duration Habitation Strategies: Architectural Approaches to Enable Human Exploration Missions," AIAA SPACE 2015 Conference and Exposition, No. AIAA 2015-4514, American Institute of Aeronautics and Astronautics, Pasadena, CA, Aug 2015.

${ }^{13}$ Reysa, R. P., Lumpkin, J. P., El Sherif, D., Kay, R., and Williams, D. E., "International Space Station (ISS) Carbon Dioxide Removal Assembly (CDRA) Desiccant/Adsorbent Bed (DAB) Orbital Replacement Unit (ORU) Redesign," No. 200701-3181, Jul 2007.

${ }^{14}$ Sherif, D. E. and Knox, J. C., "International Space Station Carbon Dioxide Removal Assembly (ISS CDRA) Concepts and Advancements," 35th International Conference on Environmental Systems, No. 2005-01-2892, Society of Automotive Engineers International, Rome, Italy, Jul 2005.

${ }^{15}$ Carter, L., "Status of the Regenerative ECLS Water Recovery System," 40th International Conference on Environmental Systems, No. AIAA 2010-6216, American Institute of Aeronautics and Astronautics, Barcelona, Spain, 2010. 
${ }^{16}$ Carter, L., Pruitt, J., Brown, C. A., Schaezler, R., and Bankers, L., "Status of ISS Water Management and Recovery," 45th International Conference on Environmental Systems, No. ICES-2015-073, International Conference on Environmental Systems, Bellevue, WA, 2015.

${ }^{17}$ Bagdigian, R. M., Dake, J., Gentry, G., and Gault, M., "International Space Station Environmental Control and Life Support System Mass and Crewtime Utilization In Comparison to a Long Duration Human Space Exploration Mission," 45th International Conference on Environmental Systems, No. ICES-2015-094, International Conference on Environmental Systems, Bellevue, WA, 2015.

${ }^{18}$ Vitali, R. and Lutomski, M. G., "Derivation of Failure Rates and Probability of Failures for the International Space Station Probabilistic Risk Assessment study," Probabilistic Safety Assessment and Management, Springer London, London, 2004, pp. 1194-1199.

${ }^{19}$ Stromgren, C., Goodliff, K., Cirillo, W., and Owens, A., "The Threat of Uncertainty - Why Using Traditional Approaches for Evaluating Spacecraft Reliability Are Insufficient for Future Human Mars Missions," AIAA SPACE 2016 Conference 83 Exposition, American Institute of Aeronautics and Astronautics, Long Beach, CA, 2016. (Submitted for publication)

${ }^{20}$ Owens, A. C. and de Weck, O. L., "Limitations of Reliability for Long-Endurance Human Spaceflight," AIAA SPACE 2016 Conference 83 Exposition, American Institute of Aeronautics and Astronautics, Long Beach, CA, 2016. (Submitted for publication)

${ }^{21}$ Sherbrooke, C. C., Optimal Inventory Modeling of Systems: Multi-Echelon Techniques, Vol. 72 of International Series in Operations Research \& 3 Management Science, Kluwer Academic Publishers, Boston, MA, second ed., 2004.

${ }^{22}$ Owens, A. C. and de Weck, O. L., "Increasing the Fidelity of Maintenance Logistics Representation in Breakeven Plots," 46th International Conference on Environmental Systems, No. ICES-2016-344, International Conference on Environmental Systems, Vienna, Austria, 2016.

${ }^{23}$ Jones, H. W., "Common Cause Failures and Ultra Reliability," 42nd International Conference on Environmental Systems, No. AIAA-2012-3602, American Institute of Aeronautics and Astronautics, San Diego, CA, 2012.

${ }^{24}$ Bodiford, M., Ray, J., Gilley, S., Kennedy, J., and Howard, R., "Are We There Yet?....Developing In-situ Fabrication \& Repair Technologies to Explore and Live on the Moon and Mars," 1st Space Exploration Conference: Continuing the Voyage of Discovery, No. AIAA-2005-2624, American Institute of Aeronautics and Astronautics, Orlando, FL, Jan 2005.

${ }^{25}$ Taminger, K. M. B., Hafley, R. A., and Dicus, D. L., "Solid Freeform Fabrication: An Enabling Technology for Future Space Missions," International Conference on Metal Powder Deposition for Rapid Manufacturing, No. Invited Keynote Lecture, Metal Powder Industries Federation, San Antonio, TX, 2002.

${ }^{26}$ Campbell, I., "Advantages of AM for production," Wohlers Report 2015: 3D Printing and Additive Manufacturing State of the Industry, edited by T. Wohlers and T. Caffrey, Wohlers Associates, Inc., Fort Collins, CO, 2015, pp. 186-189.

${ }^{27}$ Cooper, K., McLemore, C., and Anderson, T., "Cases for Additive Manufacturing on the International Space Station," 50th AIAA Aerospace Sciences Meeting including the New Horizons Forum and Aerospace Exposition, No. AIAA-2012-0517, American Institute of Aeronautics and Astronautics, Nashville, TN, Jan 2012.

${ }^{28}$ Snyder, M. and Dunn, J., "3D Printing on the International Space Station: A Technology Demonstration Paving the Way for Space Manufacturing," 65th International Astronautical Congress, No. IAC-14.A2.7.13.x27029, International Astronatical Federation, Toronto, 2014, pp. 13-15.

${ }^{29}$ Edmunson, J. E. and McLemore, C. A., "In Situ Manufacturing is a Necessary Part of Any Planetary Architecture," Concepts and Approaches for Mars Exploration, No. M12-1867, Houston, TX, 2012.

${ }^{30}$ McLemore, C., Fikes, J., Darby, C., Good, J., and Gilley, S., "Fabrication Capabilities Utilizing in Situ Materials," AIAA SPACE 2008 Conference 83 Exposition, No. AIAA-2008-7854, American Institute of Aeronautics and Astronautics, San Diego, CA, Sep 2008.

${ }^{31}$ Dunn, J. J. and Snyder, M., "Reducing Earth Dependency for Human Spaceflight Through Robotic Space Manufacturing," 65th International Astronautical Congress2, No. IAC-14,A5,3-B3.6,1,x27023, International Astronatical Federation, Toronto, Canada, 2014.

${ }^{32}$ Grenouilleau, J. C., Housseini, O., and Pérès, F., "In-Situ Rapid Spares Manufacturing and Its Application to Human Space Missions," Space 2000, American Society of Civil Engineers, Albuquerque, NM, Seb 2000, pp. 42-48.

${ }^{33}$ Niehe, P., "3D makeover for hyper-efficient metalwork," 2015. URL: http://www.arup.com/news/2015_05_may/11_may_ 3d_makeover_for_hyper-efficient_metalwork [cited 02 Aug 2016].

${ }^{34}$ McMahan, T., "Successful NASA Rocket Pump Tests Pave Way for 3-D Printed Demonstrator Engine," 2015. URL: http://www.nasa.gov/centers/marshall/news/news/releases/2015/ successful-nasa-rocket-fuel-pump-tests-pave-way-for-3-d-printed-demonstrator-engine.html [cited 08 Aug 2016].

${ }^{35}$ Joshi, S. C. and Sheikh, A. A., "3D printing in aerospace and its long-term sustainability," Virtual and Physical Prototyping, Vol. 10, No. 4, Oct 2015, pp. 175-185.

${ }^{36}$ Emerit, A., Close, J., Gavroy, G., Heinrich, R., Pikelj, M., and Lechon, F., "Airbus Defence and Space optimising components using 3D printing for new Eurostar E3000 satellite platforms," 2015. URL: https://airbusdefenceandspace.com/newsroom/news-and-features/ airbus-defence-and-space-optimising-components-using-3d-printing-for-new-eurostar-e3000-satellite-platforms/ [cited 08 Aug 2016].

${ }^{37}$ Hillier, F. S. and Lieberman, G. J., Introduction to Operations Research, McGraw-Hill Higher Education, New York, 7th ed., 2001.

${ }^{38}$ Lopez, P., Schultz, E., Mattfeld, B., Stromgren, C., and Goodliff, K., "Logistics needs for potential deep space mission scenarios post Asteroid Redirect crewed Mission," 2015 IEEE Aerospace Conference, IEEE, Big Sky, MT, Mar 2015, pp. 1-10. 